\title{
INVARIANT CHARACTERISTICS OF A POINTING MOVEMENT IN MAN ${ }^{1}$
}

\author{
J. F. SOECHTING ${ }^{2}$ AND F. LACQUANITI
}

Laboratory of Neurophysiology, University of Minnesota Medical School, Minneapolis, Minnesota 55455

\begin{abstract}
Simple arm movements involving forward projection of the hand toward a target were studied by measuring simultaneous wrist position in three-dimensional space and changes in elbow angle. An attempt was made to identify those features of the movement which exhibit invariant characteristics under the hypothesis that such invariances may reflect the operations by which central processes participate in the organization of the movement. The first such invariance to be identified was that the trajectory in space is independent of movement speed. Secondly, the movement can be viewed as consisting of two phases, an acceleratory phase and a deceleratory one, with the movement during the acceleratory phase being so organized as to maintain the ratio of elbow angular velocity to shoulder angular velocity invariant with respect to target location in the deceleratory phase. It is suggested that proprioceptive information is used to control the movement and that the latter invariance may result from a negative feedback of force involving tendon organ afferents.
\end{abstract}

Learned movements have been characterized by aspects such as the reaction time to their initiation, speed and accuracy and the inter-relationships between these factors and target size and location (cf., Fitts, 1954; Glencross, 1977; Keele, 1968; Prablanc et al., 1979; Woodworth, 1899). By contrast, relatively little work has been done on the manner in which even simple skilled movements are executed in three-dimensional space (Beggs and Howarth, 1972; Cavanagh and Landa, 1977; Georgopoulos et al., 1980; Terzuolo and Viviani, 1980; Viviani and Terzuolo, 1981). In particular, their trajectories and the relationships between the extrinsic coordinates describing the trajectory in extrapersonal space and the intrinsic coordinates of the body (changes in joint angles) as well as the sequences of muscular activity necessary for producing them are little understood even though one may reasonably expect to find stereotyped patterns which characterize some elements of the execution of a particular movement. If the invariant aspects of these patterns can be identified, one may be able to deduce some of the logical operations by which central processes participate in the organization and/or control of skilled movements.

This work was supported by United States Public Health Service Grant NS-15018. We thank Drs. E. Y. S. Chao and J. R. Dufresne for their valuable technical suggestions and R. E. Poppele and C. A. Terzuolo for a critical reading of the manuscript.

${ }^{2}$ To whom correspondence should be addressed at Laboratory of Neurophysiology, 5-257 Millard Hall, University of Minnesota Medical School, Minncapolis, MN 55455.
Such an approach has been used successfully to study complex movements, such as locomotion, which are automatic in nature. It has been shown that the duration of the swing phase of the step cycle is invariant with the speed of locomotion and that the onset of activity in all of the physiological extensors of a given leg is approximately simultaneous (Engberg and Lundberg, 1969; Grillner, 1975; Shik and Orlovsky, 1976). Invariant relations also have been proposed to account for interlimb coordination in locomotion, and consistent patterns of activation of functional syngergists have been identified in tasks involving postural stabilization (Nashner, 1977).

We have attempted a parallel approach to study a simple movement of the arm involving forward projection of the hand in extrapersonal space as in a pointing or reaching task. Such a movement requires simultaneous forward flexion at the shoulder and extension at the elbow. We shall show that several characteristics of this movement are invariant. The trajectory of the wrist is independent of the speed with which the movement is executed, and there exist invariant relations between the rates of shoulder flexion and elbow extension, especially in the terminal phases of the movements. These results raise the question of whether the movement is organized in terms of the intrinsic coordinates of the body and they lead to specific hypotheses regarding the manner in which the movement is planned and controlled.

\section{Materials and Methods}

The task. The experimental setup is schematically illustrated in Figure 1A. Each subject was instructed to 
perform a pointing movement with his arm so as to touch one of four targets presented on a television screen with his index finger, with the movement initiated from a standard position: upper arm approximately vertical, forearm horizontal, and index finger extended. The targets were arranged so that the movement was performed in the sagittal plane; the targets (which measured 2.5 by $2.0 \mathrm{~cm}$ ) were arranged vertically and equally spaced (separation $7.5 \mathrm{~cm}$ ). They were constantly in view. The subjects were directed to point to a specified target upon hearing the command, which was an audible tone. An experimental session consisted of 20 to 60 such movements with random target selection. The subjects were asked to vary the speed of their movements but were given no other instructions, regarding either the manner in which the movement was to be performed or the accuracy which was required of them.

Recording system. The changes in the elbow angle were measured goniometrically. The goniometer, which was constructed according to a design provided by Dr. E. Y. S. Chao (Biomechanics Laboratory, Mayo Clinic, Rochester, MN) has three degrees of freedom, two at the elbow and one at the wrist. Those at the elbow correspond to flexion-extension ( $\phi$ in Fig. $1 A$ ) and carrying angle (rotation of the forearm about the axis of the upper arm; cf., Morrey and Chao, 1976). Since changes in the carrying angle during the movement never exceeded $5^{\circ}$ in amplitude, this parameter will not be considered in this paper nor will changes in wrist rotation (since proper orientation of the wrist is not essential to the task). The goniometer was strapped to the upper arm and clamped to the forearm proximal to the wrist, with the potentiometer, $p$ (Fig. $1 A$ ), which measured elbow extension, being aligned with the olecranon process.

Given the complex nature of the shoulder joint, forward flexion of the arm involves a translation at the joint as well as a rotation about it (cf., Dempster, 1965; Dvir and Berme, 1978). Therefore, goniometric methods are less well suited to measure movement about the shoulder
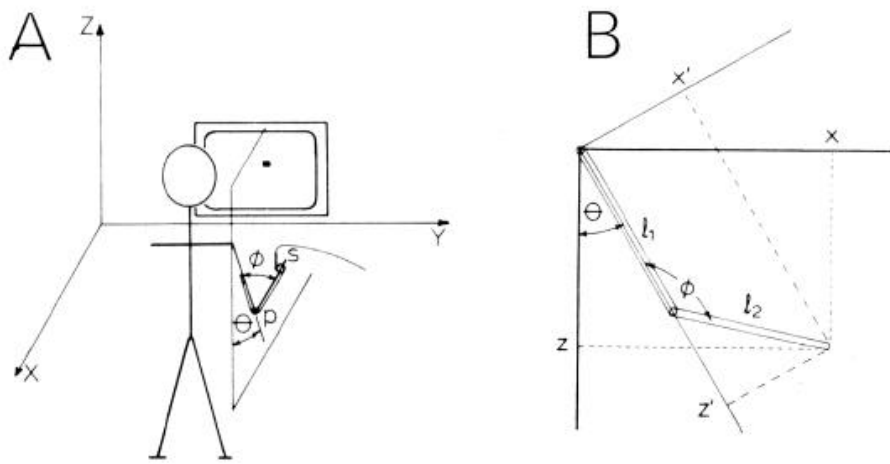

Figure 1. Schematic of experimental setup. Arm movements were executed in the sagittal plane to a target displayed on a television screen. Wrist position in three-dimensional coordinates $(X, Y, Z)$ was measured by ultrasound; the source $(s)$ is located at the wrist. Elbow angle $(\phi)$ was measured by means of a potentiometer $(p)$. Shoulder angle $(\theta)$ was calculated indirectly, using the geometrical relationships between $x, z$ and $x^{\prime}, z^{\prime}$ depicted in $B$. The length of the upper arm is $l_{1}$ and that of the forearm is $l_{2}$. joint and we resorted to indirect methods to estimate the angle of forward flexion, $\theta$ (Fig. $1 A$ ), by measuring the position of the wrist in three-dimensional space. This was accomplished by means of an ultrasound-emitting device (Graf Pen, Science Accessories Corp.) which was attached at the wrist ( $s$ in Fig. $1 A$ ). (For technical reasons, finger position could not be monitored and, thus, movement accuracy could not be assessed precisely.) The distance from the source $s$ to three orthogonal linear microphones $(X, Y, Z$ in Fig. $1 A)$ thus was measured with a resolution of $0.1 \mathrm{~mm}$ at a sampling rate of $100 \mathrm{~Hz}$.

Electromyographic activity of deltoid and biceps muscles was recorded by means of surface electrodes.

Analysis. In order to simplify the subsequent analysis and reduce the number of degrees of freedom of the movement to two (one at the shoulder and one at the elbow), movements were limited to the sagittal plane ( $y$ $=$ constant) and the amount of shoulder flexion was calculated indirectly. It was assumed that the movement proceeded in the sagittal plane (as planned) and that it consisted of pure rotation about the shoulder and elbow. If so, the angle $\theta$ can be calculated by the following procedure. Let $x$ and $z$ be the distance from the center of rotation at the shoulder to the ultrasound source, measured in the fixed $X, Z$ coordinate system (Fig. $1 B$ ). We define a primed coordinate system which rotates with the upper arm, and $x^{\prime}$ and $z^{\prime}$ represent the position of the source in this coordinate system. Since

$$
\begin{aligned}
& x^{\prime}=l_{2} \sin \phi \\
& z^{\prime}=l_{1}-l_{2} \cos \phi
\end{aligned}
$$

$x, z$ and $x^{\prime}, z^{\prime}$ are known. They are related by

$$
\begin{aligned}
& x=x^{\prime} \cos \theta+z^{\prime} \sin \theta \\
& z=z^{\prime} \cos \theta-x^{\prime} \sin \theta
\end{aligned}
$$

and solving for $\theta$

$$
\theta=\tan ^{-1} \frac{x z^{\prime}-z x^{\prime}}{x x^{\prime}+z z^{\prime}}
$$

The estimate for $\theta$ given by equation 3 is derived from both the horizontal and vertical deviation of the wrist. Each of these components taken separately (i.e., solving equation 2 for $\sin \theta$ or $\cos \theta$ ) provides another estimate of $\theta$; all three should be identically equal if the motion is a pure rotation. All three were calculated for each point of the movement as were their maximum difference and standard deviation. In 70 to $80 \%$ of the trials, the maximum difference between the estimates of $\theta$ was less than $3^{\circ}$ (with a corresponding standard deviation of about $2^{\circ}$ ). Trials in which the difference exceeded $5^{\circ}$ were discarded. Since the excursion in $\theta$ during the movements ranged from $30^{\circ}$ to $50^{\circ}$, the uncertainty in our estimation of this parameter is no more than $10 \%$.

Following double-sided exponential smoothing, angular and linear displacements were differentiated numerically to obtain the velocity and acceleration. The net torques acting at the shoulder $\left(T_{s}\right)$ and elbow $\left(T_{e}\right)$ joints required to produce the observed motion were calculated by deriving the equations of motion for the two limb 
segments according to Newton's second law:

$$
\begin{gathered}
T_{s}=\left(I_{*}+I_{e}-2 A \cos \phi\right) \ddot{\theta} \\
-\left(I_{e}-A \cos \phi\right) \ddot{\phi}-A \sin \phi \cdot \dot{\phi}^{2} \\
\quad+2 A \sin \phi \cdot \dot{\theta} \dot{\phi}+B \sin \theta-C \sin (\theta-\phi) \\
T_{e}=I_{e} \ddot{\phi}-\left(I_{e}-A \cos \phi\right) \ddot{\theta}-A \sin \phi \cdot \dot{\theta}^{2}+C \sin (\theta-\phi)
\end{gathered}
$$

where $I_{s}$ and $I_{e}$ are the moment of inertia of the upper arm (about the shoulder) and the forearm (about the elbow), respectively. The terms with coefficients $B$ and $C$ are the gravitational torques on the upper arm and forearm, respectively. $\left(B=m_{a} d_{a} g+m_{f} l_{1} g\right.$ and $C=$ $m_{f} d_{f} g$, where $m_{a}$ and $m_{f}$ are the mass of the upper arm and forearm, including hand, respectively. The coefficients $d_{a}$ and $d_{f}$ are the distance from shoulder and elbow to their respective centers of mass.) The coefficient $A$ is calculated using the equation, $A=m_{f} d_{f} l_{1}$. Values of the coefficients were calculated for each of the subjects who participated in the study on the basis of anthropometric values reported by Evans (1961) and the subjects' heights and weights. Typical values for the coefficients are: $I_{s}=$ $0.40, I_{e}=0.15, A=0.18 \mathrm{~kg}-\mathrm{m}^{2}, B=12.0$, and $C=5.0 \mathrm{~kg}-$ $\mathrm{m}^{2} / \mathrm{sec}^{2}$. The terms with coefficients $I_{s}$ and $I_{e}$ represent the torques required to produce pure angular rotation; those with angular velocity squared the centripetal acceleration of the centers of mass $\left(V^{2} / R\right)$, and those with the coefficient $A$ times angular acceleration their tangential acceleration (cf., Goldstein, 1950).

\section{Results}

The trajectory of the movement is constant and independent of velocity. Prior to the onset of the movement, each subject stood with his arm at his side (shoulder angle $\theta$, approximately equal to $0^{\circ}$ ), his forearm horizontal (elbow angle $\phi$, about $90^{\circ}$ ), and his index finger extended. Each was instructed to touch one of the targets with his index finger. Figure 2 shows the trajectories followed by the wrist during two representative trials from one subject. The targets were arranged vertically; the elevation of the target in Figure $2 A$ was greater than that in Figure $2 B$. In these instances, the range of motion in the horizontal direction $(X)$ was $42 \mathrm{~cm}$; that in the vertical direction $(Z)$ was $25 \mathrm{~cm}$ (Fig. $2 A$ ) and $6 \mathrm{~cm}$ (Fig.
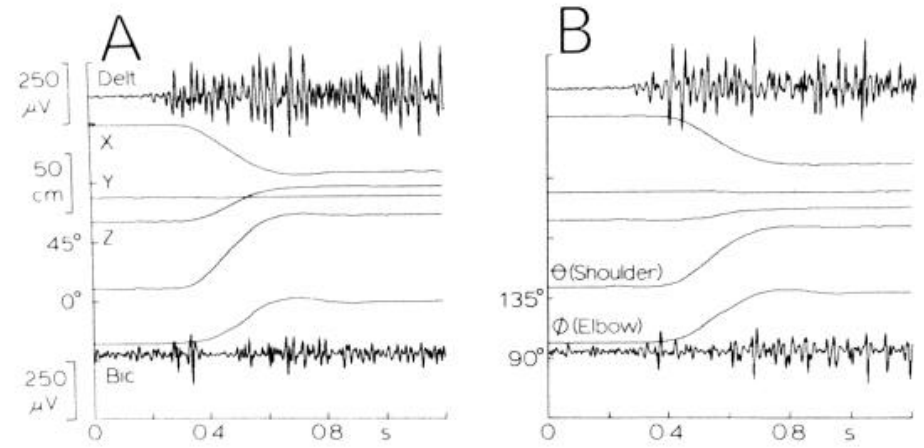

Figure 2. Representative examples of pointing movements. The traces from top to bottom depict deltoid (Delt.) EMG activity, wrist position in Cartesian coordinates, shoulder angle $\theta$, elbow angle $\phi$, and biceps (Bic.) EMG activity. The scale for $\phi$ is given in $B$. The movements are to two different targets; that in $B$ is about $20 \mathrm{~cm}$ lower than that in $A$.
$2 B$ ). Changes in wrist position in the lateral direction $(Y)$ were negligible throughout the movement in accordance with the experimental design. Therefore, it was possible to calculate the shoulder angle $(\theta)$ required to produce the instantaneous position of the wrist (equation 3 of "Materials and Methods"). The movements were accomplished by forward flexion at the shoulder of about $55^{\circ}$ (Fig. $2 A$ ) and $40^{\circ}$ (Fig. $2 B$ ) and extension at the elbow by about $28^{\circ}$ (Fig. $2 A$ ) and $32^{\circ}$ (Fig. $2 B$ ). The movements illustrated in Figure 2, having a duration of about $400 \mathrm{msec}$, are among the fastest performed by our subjects. Deltoid and biceps EMG activity also are shown in Figure 2. Before dealing with the pattern of EMG activity responsible for producing the movement, we wish to consider in detail the characteristics of the movement trajectory.

Figure 3 shows that the trajectory of the pointing movement to any given target is independent of the velocity of the movement. The results shown are from the subject who had the largest intertrial variation in speed. Parts $A$ through $D$ of Figure 3 show the movement trajectories to the uppermost target; parts $E$ through $H$ show those to a target located $15 \mathrm{~cm}$ lower. Each trace depicts the results from one trial and the arrows indicate the direction of movement. In Figure 3, $A$ and $E$, we have plotted vertical wrist position $(Z)$ versus horizontal postion $(X)$ with the movement progressing from right to left. Note that, in the extrinsic coordinates $(X, Y, Z)$, the movement follows a trajectory which is approximately a straight line, although a flattening of the slope of the trajectory is evident in Figure $3 A$ (see also Fig. $4 A$ ). The variability in the trajectory results primarily from variability in the starting position, which was not controlled precisely.

In Figure $3, B$ and $F$ show the concomitant variations in $\phi$ and $\theta$. Initially, $\theta$ is about $-10^{\circ}$ and $\phi$ is about $90^{\circ}$, and in this representation, the movement progresses from left to right. Note that, also in the intrinsic coordinates $(\theta$ and $\phi)$, the trajectory varies little from trial to trial. This should not be surprising since the transformation between the extrinsic $(X, Y, Z)$ and intrinsic coordinate representations is unique.

In Figure $3, C$ and $D$ and $G$ and $H$ are phase plane plots of the movement; that is to say, they represent plots of the angular velocity at the elbow $(\dot{\phi})$ versus angular velocity at the shoulder $(\dot{\theta})$. As plotted, the movement progresses in a counterclockwise fashion. Note that the fastest movements are almost 4 times as fast as the slowest. In the plots in Figure $3, D$ and $H$, both $\dot{\phi}$ and $\dot{\theta}$ have been normalized with respect to the maximum angular velocity at the shoulder. Despite the large difference in speed, there is very little variability among the individual traces in these plots after normalization; those which deviate the most from the mean in Figure $3 D$ are among the slowest. Once again, the invariance of these movements in the phase plane representation is a consequence of the invariance of the trajectory of the movement. Namely, if $\phi$ is uniquely related to $\theta$, then there also must be a unique relation between their angular velocities. We have chosen the phase plane representation because it most clearly shows several other features of these movements exhibited by all subjects. 

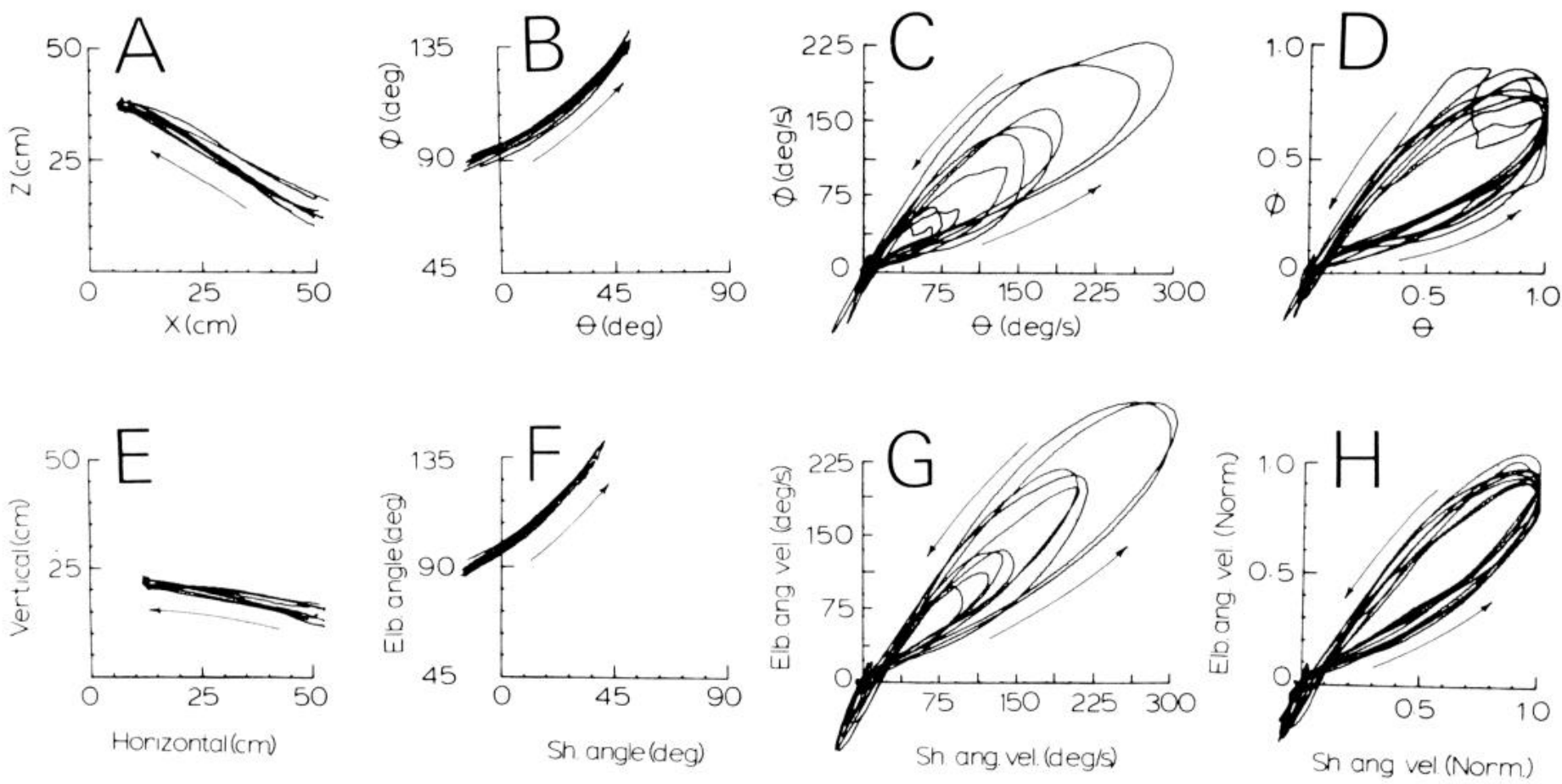

Figure 3. Description of movement trajectories. $A$ to $D$, Trajectories followed by pointing movements to one target; $E$ to $H$, those to a target $15 \mathrm{~cm}$ lower. Individual trials from one subject have been superimposed. $A$ and $E$, The trajectory of the wrist in Cartesian coordinates $(X$ and $Z$ ) with the movement progressing from right to left as indicated by the arrows; $B$ and $F$, corresponding relations between elbow $(\phi)$ and shoulder $(\theta)$ angles with the movement progressing from left to right. The relations between angular velocities at the two joints are shown in $C$ and $D$ and $G$ and $H$ with the motion progressing in a counterclockwise direction. In $D$ and $H$, angular velocities have been normalized with respect to maximum angular velocity at the shoulder. Data are from subject 6 in Table I. Elb. ang. vel., elbow angular velocity; Sh. ang. vel., shoulder angular velocity.

Firstly, the angular velocities at the shoulder $(\hat{\theta})$ and at the elbow $(\dot{\phi})$ reach their maximum at virtually the same time. For the data shown in Figure $3 D, \phi$ was maximal when $\dot{\theta}$ was at $91 \%$ of its peak value, and in Figure $3 H, \dot{\phi}$ was maximal when $\dot{\theta}$ was at $93 \%$ of its maximum. In both instances, the maximum angular velocity at the elbow lagged that at the shoulder. The average value for all of our subjects was $97 \pm 4 \%$; on average, maximal angular velocity at the elbow again lagged that of the shoulder slightly.

Figure 4 illustrates the manner in which the phase plane trajectories of $\dot{\phi}$ and $\dot{\theta}$ depend on target position. Shown in this figure are representative trials, to six different target locations, from another subject. Figure $4 A$ shows the resulting trajectories of individual trials in the extrinsic coordinate system. Initially, the subject pointed to targets numbered 1 through 4 in a random order. Then, the television screen was lowered and the sequence of trials was repeated. For this second set of trials, the subject stood closer to the screen since the extent of horizontal reach is less for the lowermost targets than for the upper ones. Targets 5 and 6 represent the lower two targets under this condition; the upper two (not plotted) coincide with targets 3 and 4 .

Figure $4 B$ shows the corresponding trajectories in the intrinsic $(\theta, \phi)$ coordinates, and their phase plane representation is given in Figure 4, $C$ to $H$. The arrows indicate the direction of the movement. Finally, the deceleratory portions (beginning from $\dot{\theta}_{\max }$ ) of all six trajectories have been superimposed in Figure 4I. The phase plane trajectories have all been normalized with respect to $\dot{\theta}_{\max }$.

Several features are apparent in these plots. Given the requirements of the task and the geometric constraints, movements to lower target positions require progressively less flexion at the shoulder, while the amount of elbow extension depends proportionately less on target location. Consequently, the ratio of the excursion in $\phi$ to that in $\theta(\Delta \phi / \Delta \theta)$ depends in a monotonic manner on target location. For the trials shown in Figure 4, these ratios are $0.81,0.90,0.95,1.12,1.32$, and 1.56 for targets 1 through 6 , respectively. The trajectories, as described in intrinsic coordinates, change accordingly.

The terminal phase of the trajectory is independent of target location. For purposes of the following description, it is most convenient to consider the phase plane representation (Fig. 4, $\mathrm{C}$ to $\mathrm{H}$ ) of the trajectories and to distinguish the acceleratory phase of the movement (up to $\dot{\theta}_{\max }$ ) from its deceleratory phase (Fig. $4 I$ ). The trajectory during the acceleratory phase depends strongly on target location. For the upper targets, when the extent of shoulder flexion exceeds that of elbow extension, angular velocity at the shoulder $(\dot{\theta})$ initially greatly exceeds $\dot{\phi}$. For example, in Figure $4 C, \dot{\phi}$ is still virtually 0 when $\dot{\theta}$ has reached $25 \%$ of its peak value. In general, the trajectories are initially curvilinear for the upper target locations (see also Figs. $3, C$ and $G$, and $5, B$ to $D$, which show data from two other subjects). As the ratio $\Delta \phi / \Delta \theta$ increases (lower targets), the acceleratory phase of the 

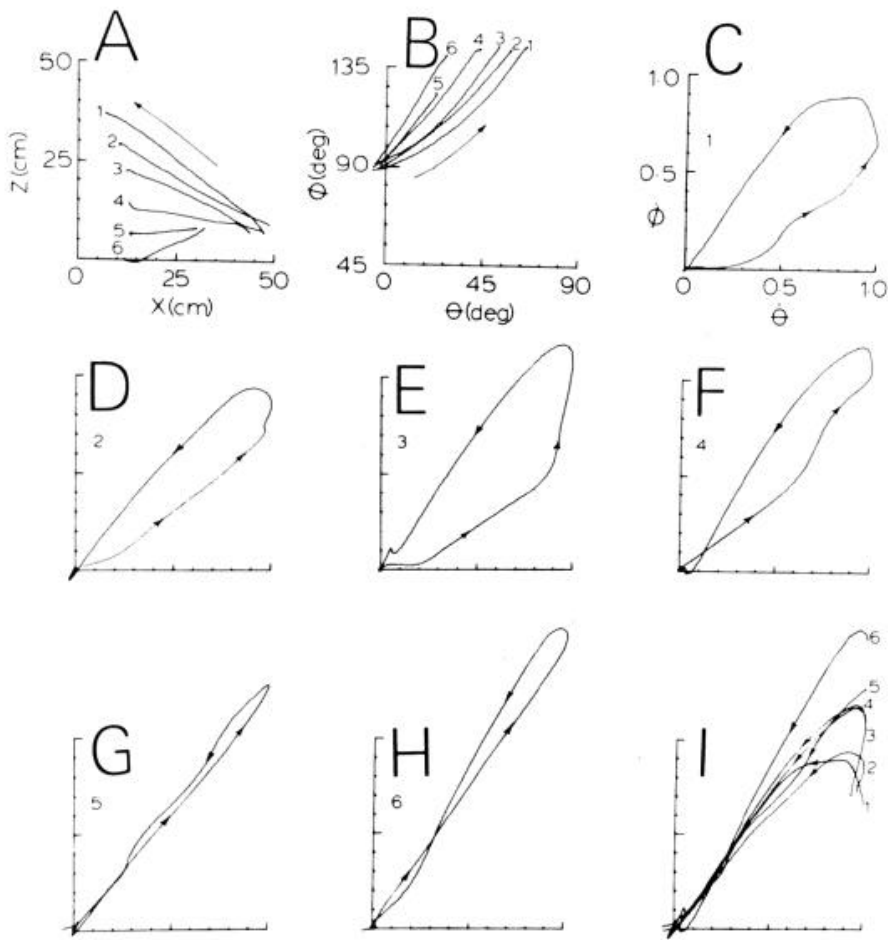

Figure 4. Dependence of movement trajectory on target location. $A$, Movement trajectories of individual trials to six target locations described in $X, Z$ coordinates; $B$, The same movements described in $\theta, \phi$ coordinates. Lower target locations require progressively less shoulder flexion. $C$ to $H$, The trajectories described in the phase plane $(\dot{\theta}, \dot{\phi})$ for movements to targets 1 to 6 , respectively. Only that portion beginning when shoulder angular velocity is maximal is shown in $I$. Note that the trajectories form a loop which becomes progressively tighter for lower target locations and that the terminal portions of all trajectories are virtually superimposable. Data are from subject 3 in Table I.

trajectory becomes progressively more rectilinear (Figs. 4, $G$ and $H$, and $5, F$ to $H$ ). In other words, for the upper targets, the slope of $\dot{\phi} / \dot{\theta}$ (and therefore the ratios of their angular accelerations) increases as the movement progresses, while it remains more constant for the lower targets.

By contrast, the phase plane trajectories are much more rectilinear during the deceleratory phase. Furthermore, and more importantly, they become virtually independent of target location as the target is approached. This is best appreciated in Figure 4I, where results from individual trials have been superimposed. Note that, with only one exception, the phase plane trajectories all converge to the same final slope, and after $\dot{\theta}$ has decreased to $40 \%$ of its maximal value, the plots are virtually superimposable. The trajectory which deviates the most from the others is for the movement toward target 4 , for which there is evidence of a corrective action (perhaps due to visual feedback) as the target is approached, as suggested by the upward curl of its trajectory in $X, Z$ coordinates (Fig. $4 A$ ) and the change in slope in $\theta, \phi$ coordinates (Fig. $4 B$ ) at the end of the movement.

Figures 5 and 6 illustrate data obtained from another subject. In Figures $5 A$ and $6 A$ are shown the trajectories (in extrinsic coordinates) of five representative move- ments to each of seven target locations. (The experiments involving target locations 1 to 4 and 5 to 7 were performed on different days; for the latter experiment, the sonic source was located slightly more proximal.) In Figure $5, B$ to $H$ show the trajectories for all of the movements performed by the subject to each of the target locations plotted in the phase plane. The corresponding plots in Figure 6 ( $B$ to $H$ ) show their mean $( \pm 1 \mathrm{SD})$ for the acceleratory phase of the movement (upper half of each panel) and its deceleratory phase (lower half).

These figures confirm the results illustrated in Figure 4 in a second subject. For the upper targets, the trajectories form a loop, with angular velocity at the shoulder $(\theta)$ initially exceeding that at the elbow. As the elevation of the target decreases, the loop becomes tighter and the angular velocities tend to become related more linearly both in the acceleratory and in the deceleratory phases. Furthermore, the slope of the angular velocities tends toward the same value in the terminal phase of the movement (after $\dot{\theta}$ has decreased to less than $25 \%$ of maximum), irrespective of target location. Moreover, in this subject, this slope is close to unity; that is to say $\dot{\phi}$ and $\dot{\theta}$ change by equal amounts as the target is approached. (A $45^{\circ}$ line is provided for reference in Figs. 5, $B$ to $H$, and $6, B$ to $H$.) Finally, as panels $I$ to $L$ in Figure 6 show, the slopes of the trajectories in the acceleratory and in the deceleratory phases of the movement do depend on target location when the trajectories are described in the extrinsic coordinate system. These plots depict the vertical velocity at the wrist $(\dot{z})$ plotted as a function of horizontal velocity $(\dot{x})$ after both variables had been normalized with respect to maximum horizontal velocity.

Table I summarizes the results obtained from all seven subjects. Target locations are listed in descending order of elevation in the first column. The values reported in subsequent columns are the mean (and standard deviation) calculated from all trials performed by each subject to each target location. The next two columns list the ratio of the change in angular extension at the elbow $(\Delta \phi)$ to that in forward flexion at the shoulder $(\Delta \theta)$ and the ratio of their maximum angular velocities. Both ratios increase monotonically with decreasing target elevation. Furthermore, there is a strong correlation between the two ratios. The last two columns provide two estimates of the asymptotic slope of the deceleratory portion of the movements. The first was calculated by fitting a straight line to individual phase plane trajectories over the interval $\theta$ greater than $10 \%$ and less than $25 \%$ of its maximal value. (There may be abrupt changes in slope for $\dot{\theta}<10 \%$ as in Fig. $4, E$ and $F$ ). The last column shows the ratio of $\dot{\phi}$ to $\dot{\theta}$ calculated when $\dot{\theta}$ was at $25 \%$ of maximum. The slope, calculated according to either method, depends little on the target position or on the relative excursions at the shoulder or elbow required to produce the movement. ${ }^{3}$ For example, for subject $2, \Delta \phi / \Delta \theta$ changes almost

${ }^{3}$ Generally, there is good agreement between the two estimates of the slope provided in Table I. The largest discrepancy occurs for target locations 5 and 6 for subject 1 , where the second estimate is significantly smaller. In these trials, $\dot{\phi}$ consistently became 0 or negative, while $\dot{\theta}$ was still positive, as is also the case for the trial shown in Figure $4 F$. 

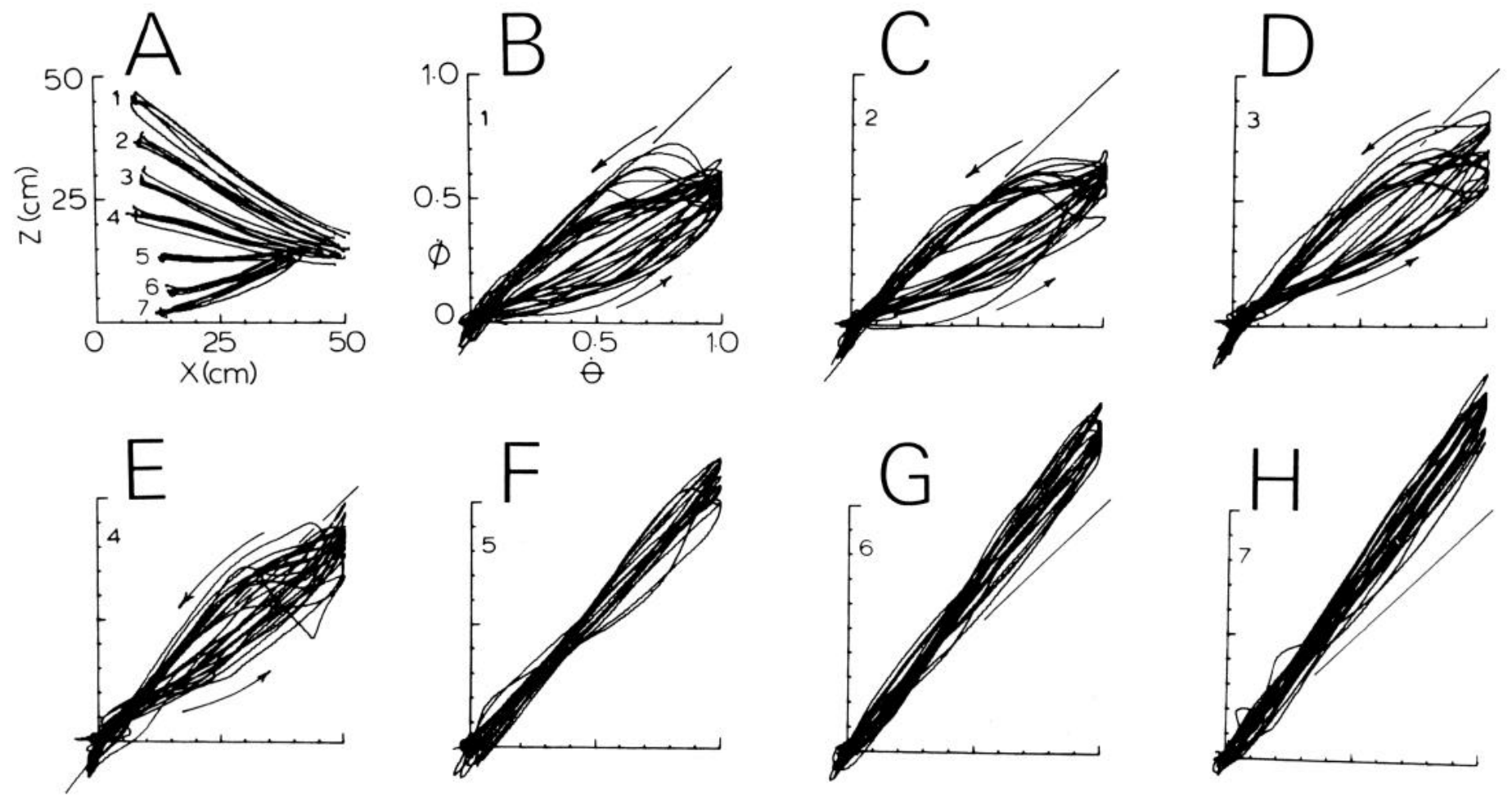

Figure 5. Dependence of movement trajectory on target locations. A, Five representative trials from one subject to each of seven different target locations; $B$ to $H$, The phase plane representation of all of the data from that subject to each of the target locations. Arrows indicate the direction of movement and a $45^{\circ}$ line $(\dot{\phi}=\dot{\theta})$ has been drawn for reference. Data are from subject 2 in Table I.

3-fold between locations 1 and 7, while the mean ratio of $\Delta \dot{\phi} / \Delta \dot{\theta}$ changes only $25 \%$. There is slightly less consistency in the values of the slopes between subjects, with mean values among subjects ranging from 0.96 to 1.36 . Despite this variability, it appears fair to conclude that the terminal slope of the trajectory as described in the phase plane of the intrinsic variables is close to or slightly exceeds unity. We defer a consideration of the possible implications of this observation on the movement to the discussion.

EMG activities and torque required to produce the movement. Figure 7 shows the average deltoid and biceps EMG activities, shoulder angle $(\theta)$, and elbow angle $(\phi)$ for movements to two target locations (1 in Fig. $7 A$ and 7 in Fig. $7 B$ ). The averages shown are those of the fastest movements this subject produced. Prior to the movement, there is little activity in the deltoid but a substantial amount in the biceps. The latter is required to counteract the force of gravity. As the movement commences, activity in the deltoid increases substantially with evidence of an overshoot. As the final position is maintained, deltoid activity remains elevated as required to counteract the force of gravity acting on the upper arm.

The biceps EMG shows a more complex pattern of activity which is strongly dependent on target position. Considering first movements to upper target locations, such as shown in Figure $7 \mathrm{~A}$, one finds a large burst of biceps activity which coincides with the increase in deltoid activity. Following this burst, biceps activity decreases, followed by an increase to a plateau. For movements to lower target positions (Fig. $7 B$ ), the initial biceps burst is lacking and activity increases more slowly and monotonically.

This pattern of biceps activity agrees well with the forces required to produce the observed trajectories to different target locations. As shown (for example, in Figs. $4 C$ and $5 B$ ), for movements to higher target locations, there is little extension at the elbow $(\dot{\phi}<\dot{\theta})$ initially and the forearm is rotated in space in the direction of flexion (the angle the forearm makes with the vertical being equal to $\phi-\theta$ ). In other words, the initial burst of biceps activity seen in Figure $7 A$ acts to retard the extension of the forearm by inertial torques. It also may assist in shoulder flexion (Basmajian, 1978). The subsequent decrease in biceps activity permits passive extension of the forearm under the force of gravity. For movements to lower target locations, $\dot{\phi}$ is initially greater than or equal to $\dot{\theta}$ (Figs. $4 H$ and $5 H$ ), and relative to the extrinsic coordinates, the forearm rotates initially in the extensor direction. The more gradual increase in biceps activity for this movement thus is to be expected.

Figure 8 shows that the patterning of deltoid and biceps activity also depends on the speed of the movement. The movements are to a target location intermediate to those shown in Figure 7 and include slow (Fig. $8 A$ ) and fast (Fig. $8 B$ ) movements. For the faster movements, the pattern of EMG activities is intermediate to those described in Figure 7. For the slower movements, the initial burst of biceps is greatly diminished. Concomitantly, inertial forces are much smaller, and gravitational forces, tending to extend the forearm, are more important.

In one subject, we monitored triceps activity. Even in 

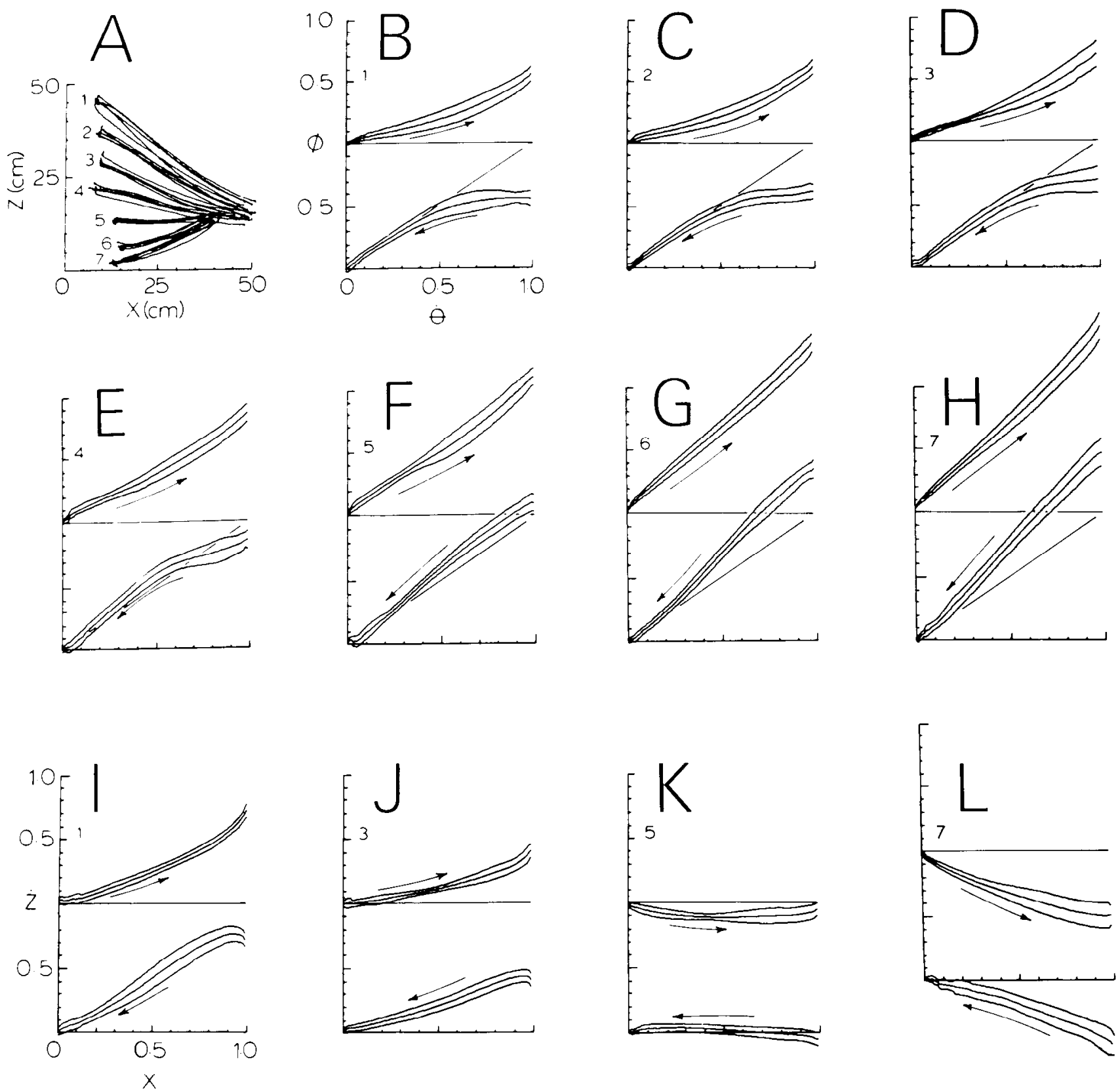

Figure 6. Dependence of acceleratory and deceleratory phases of movement on target location. Data shown are the same as those in Figure 5. $A$, Five representative trials from one subject to each of seven different target locations; $B$ to $H$, The mean $( \pm 1 \mathrm{SD})$ of the trajectories in the phase plane representation $(\dot{\theta}, \dot{\phi})$ of movements to targets 1 to 7 . The upper half of each panel shows the acceleratory phase of the movement (up to $\dot{\theta}_{\text {max }}$ ), while the lower half shows the deceleratory phase. Note that the trajectories converge onto the same slope in the deceleratory phase. Arrows indicate the direction of movement and a $45^{\circ}$ line $(\dot{\phi}=\dot{\theta})$ has been drawn for reference. $I$ to $L$, The same data represented in $\dot{x}, \dot{z}$ coordinates.

the fastest movement (Fig. $9 A$ ), very little activity was observed, indicating that the gravitational forces exceed the elastic restoring forces generated by forearm extension.

Figure 10 shows the traces of angular position $(\theta, \phi)$, velocity $(\dot{\theta}, \dot{\phi})$, and acceleration $(\ddot{\theta}, \ddot{\phi})$ at the shoulder and elbow, respectively, for the same averages shown in Figures $7 A$ and $8 B$. This figure also depicts the net torques acting at the shoulder $\left(T_{\mathrm{s}}\right)$ and at the elbow $\left(T_{e}\right)$ required to produce the movement. They were calculated according to equations 4 and 5 and they represent the total contribution by torque due to active contraction as well as due to passive viscoelastic forces acting at the shoulder and elbow. As plotted, a torque tending to flex the shoulder or the elbow assumes a positive value. The zero level for $T_{s}$ is given by the base line; that for $T_{e}$ is given by the time scale. Both $T_{s}$ and $T_{e}$ are biphasic, increasing at the onset of the movement and showing an undershoot at its arrest. Furthermore, the final steady state value of $T_{s}$ is considerably larger than its initial value, while both are approximately the same for $T_{e}$. In fact, also during the movement, there is proportionally less variation in $T_{e}$ than in $T_{s}$, especially during the deceleratory phase.

Figures 11 and 12 illustrate the manner in which $T_{s}$ 
TABLE I

Invariance of slope of angular velocities

\begin{tabular}{|c|c|c|c|c|c|}
\hline Subject & Target & $\Delta \phi / \Delta \theta$ & $\dot{\phi}_{\max } / \dot{\theta}_{\max }$ & $\Delta \dot{\phi} / \Delta \dot{\theta}$ & $\begin{array}{c}\dot{\phi} / \dot{\theta} \\
(\dot{\theta}=0.25)\end{array}$ \\
\hline \multirow[t]{6}{*}{1} & 1 & 0.61 & 0.61 & $1.05 \pm 0.06$ & $1.01 \pm 0.14$ \\
\hline & 2 & 0.63 & 0.60 & $0.91 \pm 0.08$ & $0.94 \pm 0.17$ \\
\hline & 3 & 0.68 & 0.70 & $0.76 \pm 0.15$ & $0.81 \pm 0.13$ \\
\hline & 4 & 0.80 & 0.80 & $0.91 \pm 0.17$ & $0.90 \pm 0.15$ \\
\hline & 5 & 0.79 & 0.80 & $1.03 \pm 0.14$ & $0.62 \pm 0.20$ \\
\hline & 6 & 1.05 & 1.02 & $1.11 \pm 0.17$ & $0.78 \pm 0.17$ \\
\hline \multirow[t]{7}{*}{2} & 1 & 0.52 & 0.56 & $1.02 \pm 0.14$ & $0.98 \pm 0.13$ \\
\hline & 2 & 0.63 & 0.61 & $1.04 \pm 0.12$ & $1.00 \pm 0.14$ \\
\hline & 3 & 0.72 & 0.69 & $1.08 \pm 0.13$ & $1.03 \pm 0.15$ \\
\hline & 4 & 0.85 & 0.83 & $1.24 \pm 0.13$ & $1.10 \pm 0.18$ \\
\hline & 5 & 1.09 & 1.10 & $1.23 \pm 0.11$ & $1.01 \pm 0.12$ \\
\hline & 6 & 1.32 & 1.34 & $1.28 \pm 0.14$ & $1.16 \pm 0.13$ \\
\hline & 7 & 1.40 & 1.46 & $1.28 \pm 0.18$ & $1.32 \pm 0.23$ \\
\hline \multirow[t]{6}{*}{3} & 1 & 0.70 & 0.73 & $1.31 \pm 0.16$ & $1.40 \pm 0.07$ \\
\hline & 2 & 0.82 & 0.69 & $1.13 \pm 0.15$ & $1.30 \pm 0.07$ \\
\hline & 3 & 0.89 & 0.85 & $1.15 \pm 0.27$ & $1.14 \pm 0.25$ \\
\hline & 4 & 1.02 & 1.01 & $1.30 \pm 0.22$ & $1.27 \pm 0.12$ \\
\hline & 5 & 1.28 & 1.28 & $1.19 \pm 0.29$ & $1.35 \pm 0.09$ \\
\hline & 6 & 1.65 & 1.65 & $1.44 \pm 0.17$ & $1.51 \pm 0.08$ \\
\hline \multirow[t]{4}{*}{4} & 1 & 0.52 & 0.50 & $0.89 \pm 0.07$ & $0.90 \pm 0.15$ \\
\hline & 2 & 0.59 & 0.55 & $0.95 \pm 0.12$ & $0.94 \pm 0.21$ \\
\hline & 3 & 0.72 & 0.70 & $0.94 \pm 0.08$ & $0.91 \pm 0.16$ \\
\hline & 4 & 0.94 & 0.86 & $1.17 \pm 0.19$ & $1.16 \pm 0.12$ \\
\hline \multirow[t]{4}{*}{5} & 1 & 0.55 & 0.57 & $1.13 \pm 0.16$ & $1.00 \pm 0.17$ \\
\hline & 2 & 0.67 & 0.67 & $1.21 \pm 0.14$ & $1.16 \pm 0.22$ \\
\hline & 3 & 0.84 & 0.84 & $1.11 \pm 0.17$ & $1.23 \pm 0.20$ \\
\hline & 4 & 1.04 & 1.02 & $1.19 \pm 0.22$ & $1.35 \pm 0.18$ \\
\hline \multirow[t]{4}{*}{6} & 1 & 0.73 & 0.76 & $1.33 \pm 0.13$ & $1.41 \pm 0.17$ \\
\hline & 2 & 0.79 & 0.84 & $1.32 \pm 0.13$ & $1.34 \pm 0.19$ \\
\hline & 3 & 0.88 & 0.95 & $1.35 \pm 0.15$ & $1.35 \pm 0.18$ \\
\hline & 4 & 1.01 & 1.08 & $1.43 \pm 0.15$ & $1.08 \pm 0.39$ \\
\hline \multirow[t]{4}{*}{7} & 1 & 0.87 & 0.84 & $1.16 \pm 0.12$ & $1.22 \pm 0.30$ \\
\hline & 2 & 0.77 & 0.76 & $1.16 \pm 0.22$ & $1.33 \pm 0.16$ \\
\hline & 3 & 0.92 & 0.87 & $1.16 \pm 0.10$ & $1.15 \pm 0.22$ \\
\hline & 4 & 1.00 & 1.00 & $1.22 \pm 0.26$ & $1.18 \pm 0.11$ \\
\hline
\end{tabular}
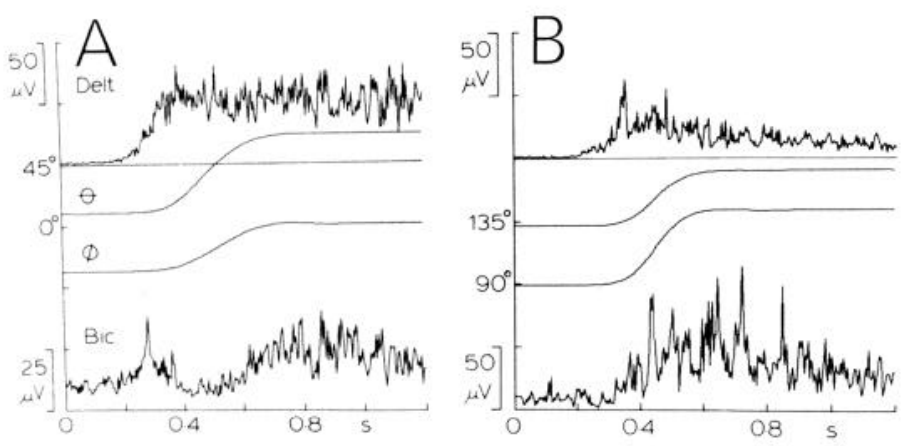

Figure 7. Description of EMG activities during the movement. Averaged deltoid (Delt.) and biceps (Bic.) EMG activity and average changes in shoulder angle $(\theta)$ and elbow angle $(\phi)$ are shown for movements to two different target locations (1 in $A$ and 7 in $B$ ). Averages are of the fastest movements that the subject produced ( 10 trials in $A$ and 4 in $B$ ) and were obtained by aligning individual trials with respect to movement onset. Note that the activity in the two muscles changes coincidently at the movement's onset but that later fluctuations in their activity do not parallel each other. The pattern of activity depends on target location. Data are from subject 2.

and $T_{e}$ depend on the velocity of the movement. Changes in torque, beginning about $200^{\circ} \mathrm{msec}$ prior to the onset of the movement, are plotted for individual trials presented in ascending order of velocity. (The data are from the
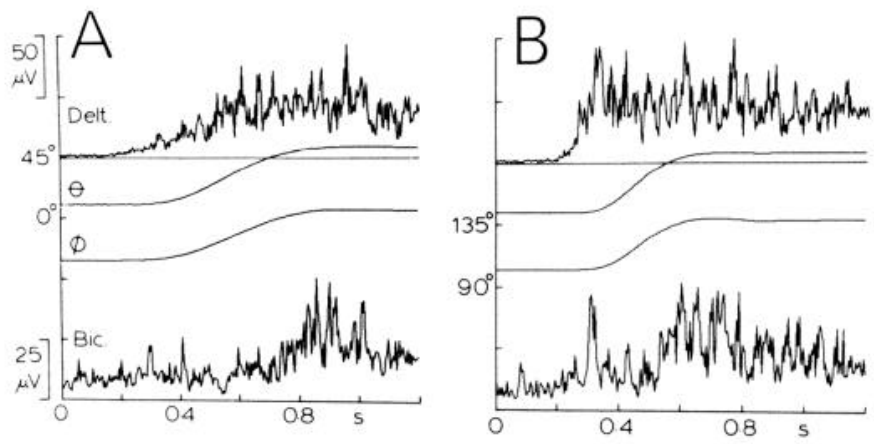

Figure 8. EMG dependence on movement speed. Traces show averaged deltoid and biceps EMG and changes in shoulder angle $(\theta)$ and elbow angle $(\phi)$ for movements to target location 4. $A$, Results for slow movements (average of 6 trials); $B$, those for fast movements (average of 6 trials). Data are from the same subject as in Figure 7.
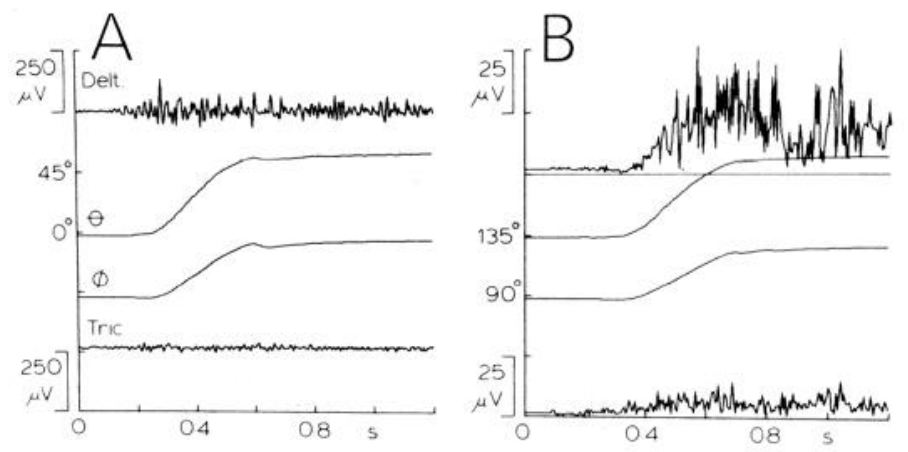

Figure 9 . Triceps EMG activity during movements. $A$, Deltoid (Delt.) and triceps (Tric.) activity during the fastest movement performed by subject 1 to target location $2 ; B$, the average of the four fastest movements to that target location.
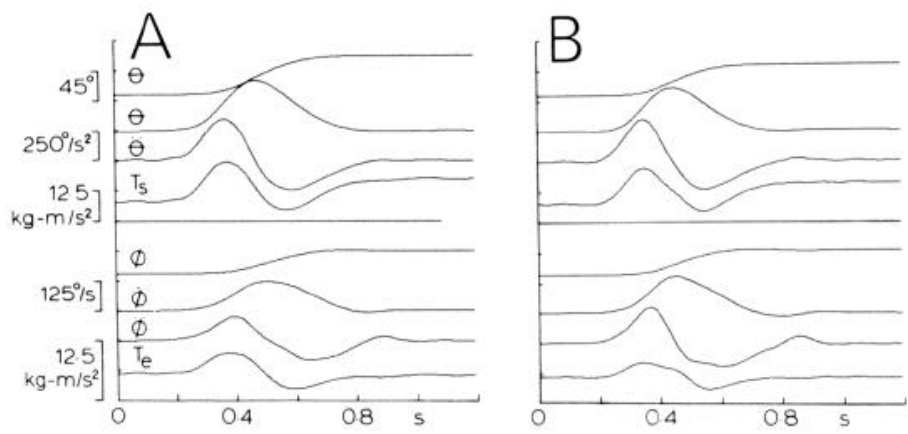

Figure 10. Torques required to produce the movement. The data are the same as those plotted in Figures $7 A(A)$ and $8 B$ $(B)$. The traces from top to bottom depict shoulder angular position, velocity and acceleration, and torque at the shoulder $\left(T_{s}\right)$ and elbow angular position, velocity and acceleration, and torque at the elbow $\left(T_{e}\right)$. As plotted, a torque tending to flex the shoulder or elbow assumes a positive sign.

two subjects in which the range of velocities was greatest.) In contrast to the invariance for the trajectories described above, there is no invariant relationship which can describe the torques required to produce the movement. In other words, the effect of velocity cannot be removed by appropriate scaling both in amplitude or in time. At low velocities, gravitational forces predominate, and $T_{s}$ increases approximately monotonically as the movement progresses, while $T_{e}$ remains virtually con- 


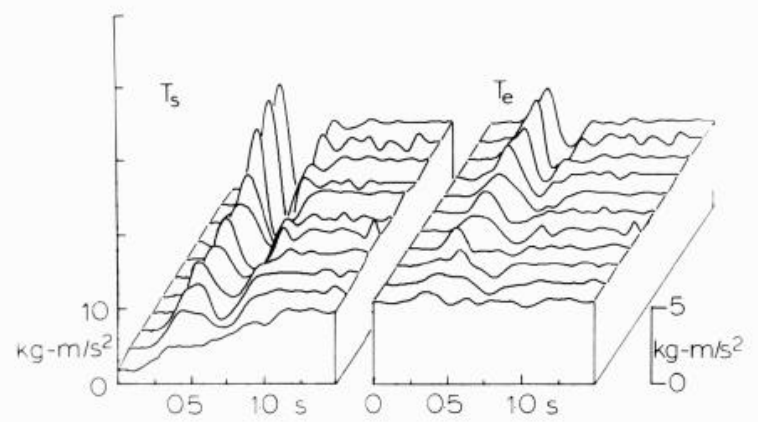

Figure 11. Dependence of torque at the shoulder $\left(T_{s}\right)$ and at the elbow $\left(T_{e}\right)$ on movement speed. Each trace depicts the calculated torque required to produce an observed movement (equations 4 and 5). The trials are plotted with equal spacing in ascending order of movement speed and are from the same subject and to the same target location.

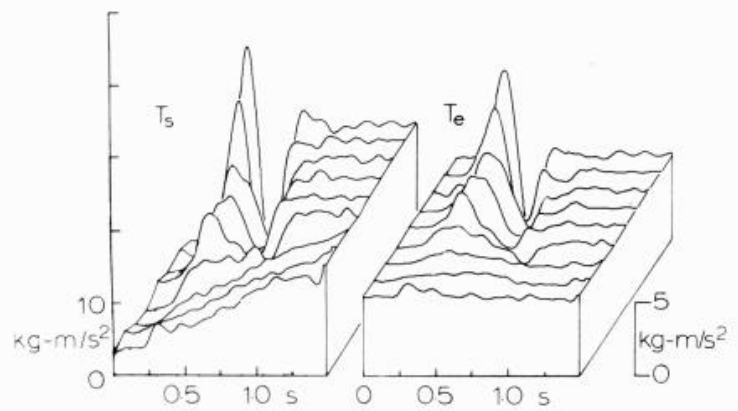

Figure 12. Dependence of torque at the shoulder and at the elbow on movement speed. Data are plotted in the same format as in Figure 11 but are from a different subject.

stant throughout. At higher velocities, inertial (angular acceleration) and Coriolis force (angular velocity squared) terms become more important and $T_{s}$ becomes progressively more biphasic as does $T_{e}$. Once again, the percentage of modulation in $T_{e}$ is much less than that of $T_{s}$.

\section{Discussion}

In this discussion, we shall focus first upon the invariances which characterize the simple, skilled movements studied and then consider some of their implications regarding the manner in which such a movement may be controlled. Before doing so, however, we wish to comment briefly upon the requirements imposed by the task and the manner in which they may have influenced the results.

In general, the requirements of the task were kept, by design, rather simple. Subjects were asked to point toward a target with a movement which, in a first approximation, involves only two degrees of freedom (elbow extension and shoulder flexion), is unidirectional, and is performed in the vertical plane. A large degree of accuracy was not required and the subjects would on occasion miss the target by as much as $0.5 \mathrm{~cm}$. Presumably then, terminal corrective actions were not a prominent feature in this task as they may be in a task requiring more accuracy (such as pushing a recessed button). Furthermore, our task did not demand a specific orientation of the hand, as may be required when a subject grasps an object or turns a handle. Finally, the task was restricted to movements requiring forward flexion at the shoulder and extension at the elbow. Thus, the question of the extent to which the invariances described in this paper can be generalized to other tasks remains open since they may depend on internal constraints imposed by the task. For example, it appears unlikely that the same set of invariances also would hold true for movements requiring elbow and shoulder flexion.

The first of these invariances is that the trajectory in space described by the movement differs little from trial to trial and is independent of the speed of the movement. This agrees with the conclusion that the trajectory described by handwriting is independent of the speed or the size of the writing (Viviani and Terzuolo, 1981). For handwriting, this result perhaps is not unexpected since the trajectory also defines the script. In our experiments, there was no such external constraint because only the initial and final positions were determined.

The fact that the trajectory of the movement does not depend on its speed implies that movement duration, and thus absolute time, is a free variable (Viviani and Terzuolo, 1981). This conclusion agrees with the finding that the pattern which characterizes the way a word is typed by professional typists is independent of the speed with which it is typed or the forces required to produce the movement (Terzuolo and Viviani, 1980). Our finding also implies that it is the trajectory and not the forces required to produce it which is invariant and which thus, by implication, is planned and controlled. In fact (Figs. 11 and 12), a movement at a given speed cannot be produced simply by an appropriate scaling in amplitude and time of the requisite torques acting at the shoulder and elbow. The net torque acting at each joint is a combination of gravitational torques (related to angular displacement and independent of speed), Coriolis forces (proportional to the square of angular velocity), and inertial torques (proportional to angular acceleration).

The other consistent findings which characterize the pointing movement examined can be summarized as follows: (1) The ratio of the maximal velocity at the elbow to that at the shoulder is equal to the ratio of the angular excursion at the two joints, (2) the two angular velocities reach a maximum at the same time, and (3) their slope is independent of target location as the target is approached. These invariances are all expressed in intrinsic coordinates and, thus, one may pose the question: is the movement organized in terms of its intrinsic coordinates $(\theta$ and $\phi)$ rather than in terms of the extrinsic coordinates $(X, Z)$ ? This would imply the existence of a coordinate transformation between the two frames of reference if the location of the target is mapped psychophysically in some extrinsic coordinate system. Recently, Pellionisz and Llinas (1980) presented a theoretical discussion pointing to the need for such transformations and suggested that they are accomplished by the cerebellum.

Note that, given the invariances mentioned above, the fact that the trajectories described in extrinsic coordinates are approximately rectilinear may be coincidental. In fact, they are more accurately described as curvilinear with the degree of curvature depending on target location (Fig. 4, $A$ and $B$ ). Also, the finding that the final slope of the trajectory described in the phase plane $(\dot{\theta}, \dot{\phi})$ is 
invariant of target location suggests that the movement can be viewed most conveniently in terms of an acceleratory and a deceleratory phase (Annett et al., 1958; Woodworth, 1899). The partition of the movement into two such phases may not be entirely arbitrary since the movement in the acceleratory phase may be organized in order to maintain the invariance of the trajectory in the deceleratory phase.

We now wish to speculate on the functional utility of the described invariances and their implications regarding the manner in which the pointing movements that we studied may be planned and controlled. The two main findings that we shall focus upon are that movement trajectory is independent of speed and that the slope $(\Delta \dot{\phi} / \Delta \dot{\theta})$ is constant and relatively independent of target location, over a wide range of movements, in the terminal phase of the movement. Regarding the latter observation, we note that, by introducing such a constraint $(\dot{\phi}=k \dot{\theta}$ $+c$ ), the number of degrees of freedom of the system is reduced from two to one in the terminal phase of the movement, and thereby, the complexity of the problem may be reduced (Bernstein, 1967; Greene, 1972). For example, if $k=1$ and $c=0$, the linear velocity at the wrist is the same as the linear velocity at the elbow.

Such a scheme, as well as the invariance of the movement trajectory with regard to speed, would appear to be implemented most readily if the kinematic variables $(\theta$ and $\phi$ ) or their derivatives were the regulated parameters. We shall consider this suggestion in more detail later, but first, we wish to discuss possible alternatives.

One theory which attempts to explain the manner in which movements, such as those that we studied, may be organized is based on the idea of a "motor memory" which would contain the patterns of muscle activity necessary to attain the final position at a given speed. Note that, given the observed strong dependence of EMG activities on movement speed and target location (Figs. 7 and 8), each such combination would need to be represented separately.

Another theory which has been proposed to explain the manner in which steady state limb position may be achieved derives from theoretical work hy Fel'dman (1974) and views muscle as a spring-mass system (cf., Bizzi et al., 1976; Kelso and Holt, 1980; Polit and Bizzi, 1979), with the springs possessing variable length-tension relationships. Final limb position would correspond to the equilibrium point of this hypothesized spring-mass system. In its simplest form, as postulated by Kelso et al. (1980), only the terminal position is determined by the parameters selected; movement trajectory between an initial and final position (kinematic details, in his terminology) would be completely arbitrary. Our finding that the trajectory of the movement did not vary with speed is inconsistent with his hypothesis. Polit and Bizzi (1979) instead proposed two independent processes: one specifying final position and one specifying velocity during the movement; the mode of operation of the latter was left unspecified.

We suggest that the most economical solution to this control problem compatible with the data would entail the existence of a planned trajectory which would be subject to the constraint that elbow and shoulder velocity be related to each other linearly as the final position is approached. Movement duration is simply varied by a scaling in time. If the movement then is controlled utilizing negative feedback related to the difference between the actual and intended trajectory (cf., Matthews, 1980), the need to calculate precisely the components of torque due to inertial, Coriolis, and gravitational forces for a given desired velocity is obviated. The contribution of such a negative feedback to EMG activity has been demonstrated for simple movements involving only one joint (Dufresne et al., 1980; Gottlieb and Agarwal, 1980).

The hypothesis also provides a tentative explanation for the invariant relationship observed as the target is approached. We consistently found that the resultant torque at the elbow $\left(T_{e}\right)$ was virtually constant during this phase of the movement (for example, for $t>0.7 \mathrm{sec}$ in Fig. 8), which could be achieved by a negative feedback contrul on $T_{e}$. The only receptor which is capable of signaling variations in this parameter is the Golgi tendon organ since $T_{\epsilon}$ reflects forces due to active contraction and due to the viscoelastic properties of muscle. Since there is ample evidence for a projection of Golgi tendon organ afferents to the cerebellum via the dorsal and ventral spinocerebellar tracts and their forelimb analogues (cf., Bloedel, 1973; Oscarsson, 1965) and since force has been said to be represented in the activity of units in the intermediate cerebellum which form part of a negative feedback loop of force (Giuffrida et al., 1979; Licata et al., 1979), we suggest that this mechanism could be involved in keeping the torque at the elbow constant.

Finally, we not infrequently saw abrupt changes in the slope of the trajectory in the phase plane when $\dot{\theta}$ had decreased to less than $10 \%$ of its maximal value (Fig. 4, $E$ and $F$ ), suggesting visually guided corrective actions. This hypothesis is supported by Hay (1979). He studied pointing movements by children to a target and induced movement errors by fitting the children with prismatic lenses which shifted the visual field laterally by $17^{\circ}$. He found (for 11-year-old children, who are presumably closest in performance to the adults in our study) that in $90 \%$ of the trials, a correction of the visually induced error occurred only in the last $16 \%$ of the trajectory.

\section{References}

Annett, J., C. W. Golby, and H. Kay (1958) The measurement of elements in an assembly task - the information output of the human motor system. Q. J. Fxp. Psychol. 10: 1-11.

Basmajian, J. V. (1978) Muscles Alive, p. 194, Williams and Wilkins, Baltimore, MD.

Beggs, W. D. A., and C. I. Howarth (1972) The movement of the hand towards a target. Q. J. Exp. Psychol. 24: 448-453.

Bernstein, N. (1967) The Coordination and Regulation of Movements, Pergamon, Oxford.

Bizzi, E., A. Polit, and P. Morasso (1976) Mechanisms underlying achievement of final head position. J. Neurophysiol. 39: 435-444.

Bloedel, J. R. (1973) Cerebellar afferent systems: A review. Prog. Neurobiol. 2: 1-68.

Cavanagh, P. R., and J. Landa (1977) A biomechanical analysis of the karate chop. Res. Q. 47: 610-618.

Dempster, W. T. (1965) Mechanisms of shoulder movement. Arch. Phys. Med. Rehab. 46: 49-70.

Dufresne, J. R., J. F. Soechting, and C. A. Terzuolo (1980) Modulation of the myotatic reflex gain in man during intentional movements. Brain Res. 193: 67-84. 
Dvir, Z., and N. Berme (1978) The shoulder complex in elevation of the arm: A mechanism approach. J. Biomech. 11:219225.

Engberg, I., and A. Lundberg (1969) An electromyographic analysis of muscular activity in the hindlimbs of the cat during unrestrained locomotion. Acta Physiol. Scand. 75: 614-630.

Evans, F. G. (1961) Biomechanical Studies of the Musculoskeletal System. pp. 95-97, Charles C Thomas, Springfield, IL.

Fel'dman, A. G. (1974) The control of muscle length. Biofizika 19: 749-753.

Fitts, P. M. (1954) The information capacity of the human motor system in controlling the amplitude of movement. J. Exp. Psychol. 47: 381-391.

Georgopoulos, A. P., J. F. Kalaska, and J. T. Massey (1980) Cortical mechanisms of two-dimensional aiming arm movements. I. Aiming at different target locations. Soc. Neurosci. Abstr. 6: 156.

Giuffrida, R., G. L. Volsi, M. R. Panto, V. Perciavalle, F. Santangelo, and A. Urbano (1979) Muscle input to rubrospinal neurons in the cat. Neurosci. Lett. (Suppl.) 3: S112.

Glencross, D. J. (1977) Control of skilled movements. Psychol. Bull. 84: 14-29.

Goldstein, H. (1950) Classical Mechanics, pp. 93-184, AddisonWesley Co., Reading, MA.

Gottlieb, G. L., and G. C. Agarwal (1980) Response to sudden torques about ankle in man. III. Suppression of stretchevoked responses during phasic contraction. J. Neurophysiol. 44: 233-246.

Greene, P. H. (1972) Problems of organization of motor systems. Prog. Theor. Biol. 2: 304-338.

Grillner, S. (1975) Locomotion in vertebrates: Central mechanisms and reflex interaction. Physiol. Rev. 55: 247-303.

Hay, L. (1979) Spatial-temporal analysis of movements in children: Motor programs versus feedback in the development of reaching. J. Mot. Behav. 11: 189-200.

Keele, S. W. (1968) Movement control in skilled motor performance. Psychol. Bull. 70: 387-403.

Kelso, J. A. S., and K. G. Holt (1980) Exploring a vibratory systems analysis of human movement production. J. Neurophysiol. 43: 1183-1196.

Kelso, J. A. S., K. G. Holt, and A. E. Flatt (1980) The role of proprioception in the perception and control of human movement: Toward a theoretical reassessment. Percept. Psychophys. 28: 45-52.

Licata, F., V. Perciavalle, F. Santangelo, A. Urbano, and A. Viscuso (1979) Computer simulation of cerebellar input-output motor relationships. Neurosci. Lett. (Suppl.) 3: S125.

Matthews, P. B. C. (1980) Developing views on the muscle spindle. Prog. Clin. Neurophysiol. 8: 12-27.

Morrey, B. F., and E. Y. S. Chao (1976) Passive motion of the elbow joint. J. Bone Joint Surg. (Br.) 58A: 501-508.

Nashner, L. M. (1977) Fixed patterns of rapid postural responses among muscles during stance. Exp. Brain Res. 30: $13-24$.

Oscarsson, O. (1965) Functional organization of the spino- and cuneocerebellar tracts. Physiol. Rev. 45: 495-522.

Pellionisz, A., and R. Llinas (1980) Tensorial approach to the geometry of brain function: Cerebellar coordination via a metric tensor. Neuroscience 5: 1125-1136.

Polit, A., and E. Bizzi (1979) Characteristics of motor programs underlying arm movements. J. Neurophysiol. 42: 183-194.

Prablanc, C., J. F. Echallier, E. Komilis, and M. Jeannerod (1979) Optimal response of eye and hand motor systems in pointing at a visual target. I. Spatio-temporal characteristics of eye and hand movements and their relationships when varying the amount of visual information. Biol. Cybern. 35: $113-124$.

Shik, M. L., and G. N. Orlovsky (1976) Neurophysiology of locomotor automatism. Physiol. Rev. 56: 465-501

Terzuolo, C. A., and P. Viviani (1980) Determinants and characteristics of motor patterns used for typing. Neuroscience 5: 1085-1103.

Viviani, P., and C. A. Terzuolo (1981) The organization of movement in handwriting and typing. In Language Production: II. Production of Non-Speech Modalities, B. Butterworth, ed., Academic Press, New York.

Woodworth, R. S. (1899) The accuracy of voluntary movement. Psychol. Rev. Suppl. 3 (2, Whole No. 13). 